\title{
Novel Mechanism of Conventional BODIPY Conjugates for Live-cell SMLM Reveals Lipid Droplet Biology at Super-resolution
}

\author{
Santosh Adhikari ${ }^{1}$, Joe Moscatelli ${ }^{2}$ and Elias M. Puchner ${ }^{1}$ \\ ${ }^{1}$ University of Minnesota, Twin Cities, Minneapolis, Minnesota, United States, ${ }^{2}$ Middlebury College, \\ Middlebury, Vermont, United States
}

Quantitative Single-Molecule Localization Microscopy (SMLM) is evolving into a powerful biophysical technique to study biological processes below the optical diffraction limit ${ }^{1-4}$. However, due to motion of the imaged structures during the long data aquisition times, most studies have been limited to fixed cells. In addition, it has been a challange to label hyrdophobic environments such as lipid droplets due to the properties of availlable fluorophores. A requirement for SMLM are fluorescent probes that have 1) high specificity for a subcellular structure under investigation and 2) photophysical properties that allow for the stochastic on-off switching in order to localize spatially well-separated point-spread functions of single fluorophores. Recently, we reported a novel strategy and class of fluorophores for live-cell SMLM: the imaging of sparse, red-shifted ground state dimers (DII), which transiently arise from bi-molecular encounters of conventional BODIPY dyes ${ }^{5}$. Hundreds of specific BODIPY conjugates are commercially available for conventional fluorescence microscopy and using them for SMLM in living cells is strikingly simple. This approach has other advantages such as intrinsic background suppression and an almost never depleting source of single molecule localizations. We demonstrated the advantages of DII-state SMLM by resolving the nanoscopic spatial regulation and the dynamics of single fatty acid analogs (FAs) and lipid droplets (LDs) in living yeast and mammalian cells with two colors.

Lipid droplets (LDs) are composed of a neutral lipid core surrounded by a phospholipid monolayer ${ }^{6,7}$. The catabolism and anabolism of LDs by regulatory proteins and enzymes is actively regulated to maintain the lipid homeostasis and to adapt to energy needs of a cell. When fatty acids (FAs) are availlable or in access, they are used for membrane expansion and storage in LDs across many cell types and species. Similarly, the release of FAs for energy production by the breakdown of LDs during lipolysis and lipophagy has been studied in the $\operatorname{past}^{8}$. However, since conventional fluorescence microscopy cannot resolve LDs and FA structures as well as their subcellular location below the optical diffraction limit, little is known about how the metabolic state of cells regulates the dynamics and the nanoscopic spatial distribution of fatty acids and neutral lipid. Likewise, the relation between the exact size of LDs and the density of regulatory enzymes on them in different metabolic states remains elusive.

Here, we present our DII-state SMLM approach together with new results from quantitative photoactivated localization microscopy (PALM) of mEos2-tagged proteins to resolve the nanoscopic spatial regulation and dynamics of single fatty acids (FAs), lipid droplets (LDs) and Fatty Acid Activating Proteins (FAAps) in living yeast with two colors. In fed cells, FAs localize to the endoplasmic reticulum (ER) where LDs with a neutral lipid core of $\sim 125 \mathrm{~nm}$ emerge and expand. Fatty acid activating proteins localize to the ER as well and form a significantly larger protein layer around LDs in fed conditions.

Upon fasting, LDs re-localize and get immobilized at the vacuole where few LDs enter the vacuolar lumen in the process of lipophagy, which breaks down neutral lipids and releases FAs. Importantly, our quantitative live cell PALM approach detects a significantly increased density of FAAps on the surface 
of LDs where their catalytic activity is needed to activate the released FAs. Activated FAs again get converted to neutral lipids and stored in LDs, which consequently have a significantly larger diameter of 450+/- $80 \mathrm{~nm}$ compared to LDs in fed cells. When external FAs are added to fasting cells, they exhibit a strikingly different behavior compared to fed cells. Instead of localizing to the ER and being incorporated into LDs, FAs form dense, non-LD clusters of $\sim 100 \mathrm{~nm}$ diameter at the plasma membrane and transition from free diffusion to confined immobilization. This phenomenon could be a new mechanism to protect cells from lipotoxicity, which is known to be a burden for fasting cells.

Besides gaining these new biological insights into LD, FA and FAAps distributions and dynamics, we further demonstrate the versatility and general applicability of DII-state SMLM by resolving lysosomes in mammalian cells with conventional BODIPY conjugates. The simple and versatile live-cell SMLM capability of the hundreds of available BODIPY conjugates opens up new avenues to study and resolve sub-cellular structures at the nanoscale far beyond the demonstrated applications.
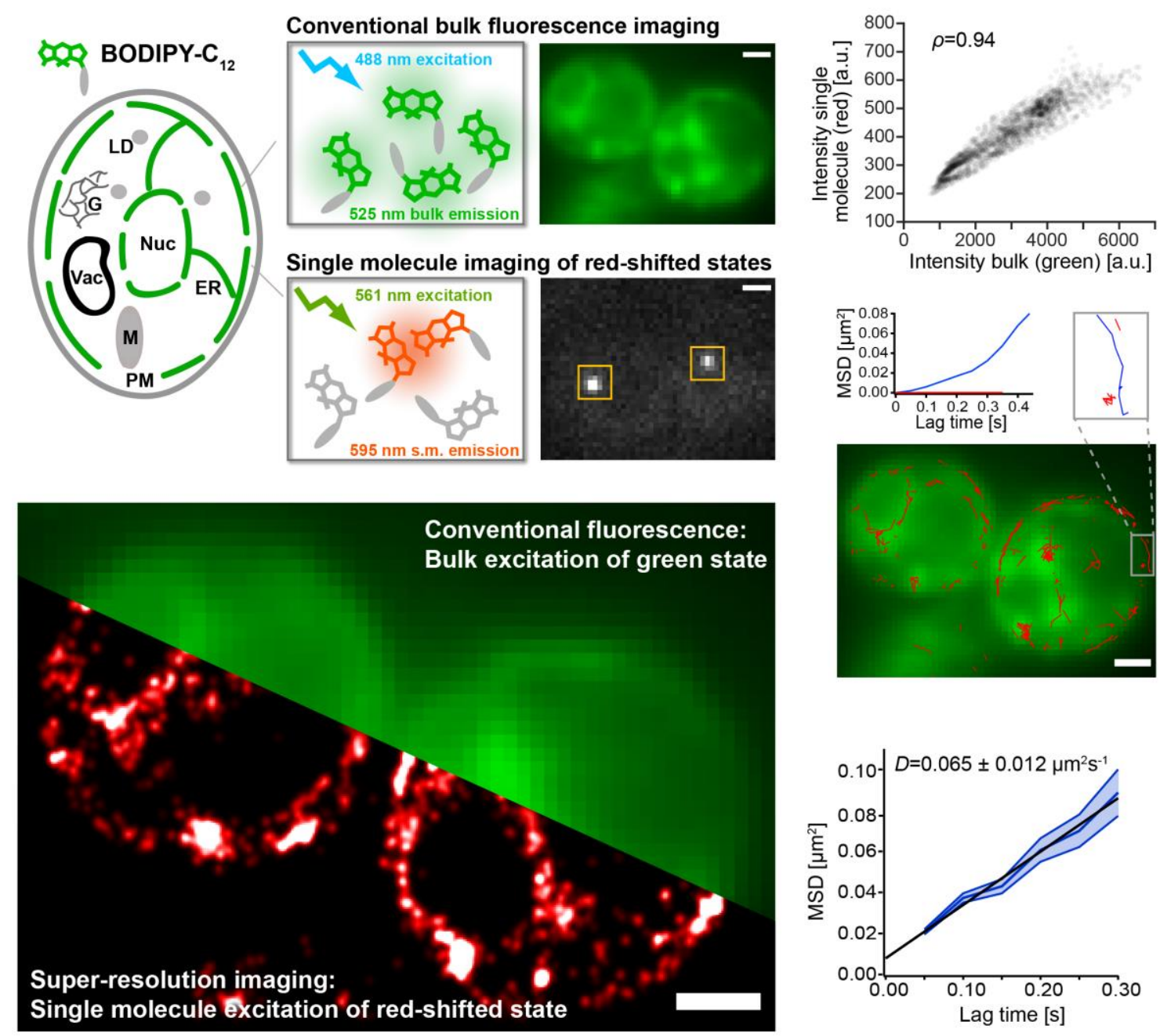

Figure 1. SMLM and tracking with transient red-shifted DII-states of conventional BODIPY conjugates. a BODIPY fluorophores exist as various conjugates that label specific compartments within cells. BODIPY-C12 is a fatty acid analog that localizes to the ER of living yeast cells when excited at $488 \mathrm{~nm}$ in the conventional fluorescence microscopy mode (green, upper). When excited at $561 \mathrm{~nm}$, bright and red-shifted single-molecule fluorescence signals appear and disappear throughout the cell (white, lower). 
b The bright single-molecule fluorescence can be used for SMLM to obtain a tenfold higher resolution image of the fatty acid distribution within the cell (red). c The pixel intensities of the conventional fluoresce image of BODIPY-C12 excited at $488 \mathrm{~nm}$ correlate well with the single-molecule signal excited at $561 \mathrm{~nm}$ and averaged over 800 frames (Pearson's $\rho=0.94$, number of cells $=2$, number of data points $=2128$ ), confirming the common origin of the two signals. $d$ The detected single-molecule signal of DII-BODIPY states lasts long enough to track fatty acid analogs (red traces). Different mobile (blue) and immobile (red) species can be discriminated. e The average mean square displacement vs. time of fatty acid analogs of 150 traces each lasting for at least 3 acquisition frames $(0.15 \mathrm{~s})$ results in an average diffusion coefficient of $0.065 \pm 0.012 \mu \mathrm{m} 2 \mathrm{~s}-1$ (error band: s.e.m., scale bars: $1 \mu \mathrm{m}$ ). Figure from Adhikari, S., Moscatelli, J., Smith, E.M. et al. "Single-molecule localization microscopy and tracking with red-shifted states of conventional BODIPY conjugates in living cells." Nat Commun 10, 3400 (2019) with permission under the Creative Commons license http://creativecommons.org/licenses/by/4.0/

\section{References}

1. Annibale, P., Vanni, S., Scarselli, M., Rothlisberger, U. \& Radenovic, A. Quantitative photo activated localization microscopy: unraveling the effects of photoblinking. PLOS ONE 6,e22678 (2011).

2. Puchner, E. M., Walter, J. M., Kasper, R., Huang, B. \& Lim, W. A. Counting molecules in single organelles with superresolution microscopy allows tracking of the endosome maturation trajectory. Proc. Natl. Acad. Sci. U.S.A.110,16015-16020 (2013).

3. Durisic, N., Laparra-Cuervo, L., Sandoval-Álvarez, A., Borbely, J. S. \& Lakadamyali, M. Singlemolecule evaluation of fluorescent protein photoactivation efficiency using an in vivo nanotemplate. Nat. Methods 11,156-162 (2014).

4. Betzig, E., Patterson, G. H., Sougrat, R., Lindwasser, O. W., Olenych, S., Bonifacino, J. S., Davidson, M. W., Lippincott-Schwartz, J. \& Hess, H. F. Imaging intracellular fluorescent proteins at nanometer resolution. Science 313,1642-1645 (2006).

5. Adhikari, S., Moscatelli, J., Smith, E. M., Banerjee, C. \& Puchner, E. M. Single-molecule localization microscopy and tracking with red-shifted states of conventional BODIPY conjugates in living cells. Nat Commun 10,1-12 (2019).

6. Tauchi-Sato, K., Ozeki, S., Houjou, T., Taguchi, R. \& Fujimoto, T. The surface of lipid droplets is a phospholipid monolayer with a unique Fatty Acid composition. J. Biol. Chem. 277,44507-44512 (2002). 7. Walther, T. C. \& Farese, R. V. Lipid Droplets And Cellular Lipid Metabolism. Annu Rev Biochem 81,687-714 (2012).

8. Rambold, A. S., Cohen, S. \& Lippincott-Schwartz, J. Fatty acid trafficking in starved cells: regulation by lipid droplet lipolysis, autophagy, and mitochondrial fusion dynamics. Dev. Cell 32,678-692 (2015). 